Check for updates

Cite this: Phys. Chem. Chem. Phys., 2019, 21, 14226

Received 8th September 2018, Accepted 29th November 2018 DOI: $10.1039 / c 8 c p 05693 e$

rsc.li/pccp

\section{Correspondence between electronic structure calculations and simulations: nonadiabatic dynamics in $\mathrm{CS}_{2} \dagger$}

\begin{abstract}
Darren Bellshaw, ${ }^{a}$ Russell S. Minns ${ }^{b}$ and Adam Kirrander (D) *a
The choice of $a b$ initio electronic structure method is an important factor in determining the fidelity of nonadiabatic dynamics simulations. We present an in-depth comparison of two simulations of photodissociation in the $\mathrm{CS}_{2}$ molecule following excitation to the $1^{1} \mathrm{~B}_{2}$ state. The simulations account for nonadiabatic and spin-orbit coupling, and are performed using the SHARC surface-hopping approach combined with state-averaged SA8-CASSCF(8,6)/SVP and SA8-CASSCF $(10,8) / S V P$ ab initio calculations, with additional reference calculations at the $\mathrm{MRCI}(14,10) /$ aug-cc-pvTZ level. The relative performance and veracity of the simulations can be assessed by inspection of the potential energy curves along specific coordinates. The simulations demonstrate direct competition between internal conversion and intersystem crossing, with strong correlation between molecular geometry, electronic state density, and dynamics.
\end{abstract}

\section{Introduction}

Photochemical processes are ubiquitous in nature as well as increasingly important for new technologies. ${ }^{1,2}$ The initial stages of photochemical dynamics occur on ultrashort timescales and generally involve rapid electronic relaxation via internal conversion mediated by nonadiabatic couplings ${ }^{3,4}$ or intersystem crossing by spin-orbit couplings. ${ }^{5,6}$ Over the past decade, remarkable progress has been made in experimental techniques capable of following the dynamics, including ultrafast spectroscopy ${ }^{7-12}$ and diffraction. ${ }^{13-19}$ Mechanistic interpretation of the experimental observations often involves comparison to simulations, making it possible to pull out features not immediately obvious from experiments alone..$^{20-25}$ There are also increasing efforts towards the calculation of observables directly from simulations, ${ }^{20,25-29}$ further strengthening the links between experiments and theory.

Given the importance of simulations for the interpretation of experiments, it is vital to consider the approximations inherent in most simulations. Numerically exact propagation of the full molecular wavefunction ${ }^{30-32}$ is not feasible in general due to the exponential scaling of the wavepacket propagation with the number of vibrational degrees of freedom in the system. Benchmark methods such as $\mathrm{MCTDH}^{33}$ are in many

\footnotetext{
${ }^{a}$ EaStCHEM, School of Chemistry, University of Edinburgh, David Brewster Road, EH9 3FJ Edinburgh, UK. E-mail: Adam.Kirrander@ed.ac.uk; Tel: $+44(0) 1316504716$

${ }^{b}$ Chemistry, University of Southampton, Highfield, Southampton SO17 1BJ, UK

$\dagger$ Electronic supplementary information (ESI) available. See DOI: 10.1039/c8cp05693e
}

cases only feasible for reduced-dimensionality models that are based on prior assumptions about the dynamics and require precalculated potential energy surfaces with significant up-front investment of computational resources. In contrast, trajectorybased methods, ${ }^{34-40}$ which calculate the electronic structure of the molecule on-the-fly, often provide sensible results at viable computational expense without resorting to reduced dimensionality models even for comparatively large molecules. These methods are therefore increasingly becoming the default choice for the interpretation of experiments..$^{720-22,25,41-43}$ However, the $a b$ initio electronic structure calculations, which ultimately constrain the fidelity of the simulations, ${ }^{44}$ remain a severe computational bottleneck. Invariably, a compromise between the ideal level of electronic structure theory for the simulations and the level commensurate with available computational resources must be struck. We set out in this article to examine how the quality of electronic structure calculations influences the dynamics observed in trajectory-based simulations, and provide a detailed analysis of the resulting dynamics.

The selected model system is the $\mathrm{CS}_{2}$ molecule, which is an important benchmark for ultrafast dynamics. Upon excitation, a complicated interplay between the nuclear and electronic motions ensues, dictated by the dense manifold of singlet and triplet electronic states that coexist in the Franck-Condon region. Important experimental studies of the $\mathrm{CS}_{2}$ molecule in the time-domain include seminal molecular-alignment and UV photoelectron imaging work, ${ }^{11,12}$ and extensive studies using photoelectron spectroscopy. ${ }^{10,45-47}$ We simulate the photodissociation dynamics of $\mathrm{CS}_{2}$ with the surface-hopping SHARC method, ${ }^{36,48,49}$ which is based on Tully's semiclassical fewest 




Fig. 1 Schematic representation of the $\mathrm{CS}_{2}$ photodissociation, showing a small subset of the potential energy surfaces included in the simulations: the molecule is excited from the ground electronic state $S_{0}\left(1^{1} A^{\prime}, 1^{1} A_{1}, \tilde{X}^{1} \Sigma_{g}^{+}\right)$ to the $S_{2}\left(2^{1} A^{\prime}, 1^{1} B_{2},{ }^{1} \Delta_{u}\right)$ state, with the main pathway to dissociation being via intersystem crossing to the triplet states which have a lower barrier to dissociation, here represented by the $\mathrm{T}_{1}\left(1^{3} \mathrm{~A}^{\prime}, 1^{3} \mathrm{~B}_{2},{ }^{3} \Sigma_{\mathrm{u}}^{+}\right)$state. For a full conversion table of electronic state labels in each point group, see Table 2.

switches algorithm ${ }^{50}$ and accounts for both nonadiabatic couplings (internal conversion) and spin-orbit coupling (intersystem crossing). This process is shown schematically in Fig. 1. Here, the molecule is excited to the $1^{1} \mathrm{~B}_{2}$ state rather than the $2^{1} \mathrm{~B}_{2}$ state of most experiments, which reduces the number of interacting states from 19 to 8 in our multiconfigurational calculations, reducing computational cost while retaining much of the complexity of the dynamics. Interestingly, many of the qualitative aspects of the dynamics bear a strong resemblance to those observed upon excitation to the higher lying $2^{1} \mathrm{~B}_{2}$ state. Previous experiments on the $1^{1} \mathrm{~B}_{2}$ state, whose absorption is about four orders of magnitude weaker than the $2^{1} \mathrm{~B}_{2}$ state at its absorption peak, have focused on photolysis and fluorescence quantum yield studies, ${ }^{51-53}$ often in the context of atmospheric chemistry. Furthermore, the high kinetic energy initial conditions limit the validity of direct comparison to experiments (see Computational details). The simulations reported here do not intend to be a direct comparison between experiment and theory, but rather an analysis of the impact of the level of theory on complex dynamics simulations.

\section{Method}

The SHARC method ${ }^{36,48,49}$ was developed to account for arbitrary couplings such as spin-orbit coupling or those induced by a laser field via a reformulation of surface-hopping in terms of an unitary transformation matrix. Electrons are treated quantum mechanically while nuclear motion is treated classically. At each time step an algorithm is followed to determine the active state to which a trajectory is localised, based on the evolution of the electronic wavefunction along the nuclear trajectory. This involves choosing a model space that covers the necessary manifold of electronic states on which the dynamics of the system will evolve. Equations-of-motion for the electronic states are determined by expansion of the electronic wavefunction and insertion into the time-dependent Schrödinger equation (see ref. 36 and 49 for derivation and a full description of the method), and may be written in compact matrix form as,

$$
\frac{\mathrm{d}}{\mathrm{d} t} \mathbf{c}^{\mathrm{rep}}=-\left[i \mathbf{H}^{\mathrm{rep}}+\mathbf{T}^{\mathrm{rep}}\right] \mathbf{c}^{\mathrm{rep}},
$$

where $\mathbf{c}^{\text {rep }}$ is the vector of wavefunction coefficients, $\mathbf{T}^{\text {rep }}$ is the temporal coupling matrix (a function of the nonadiabatic coupling matrix $\mathbf{K}^{\text {rep }}$ ), and $\mathbf{H}^{\text {rep }}$ the Hamiltonian matrix. The superscript 'rep' refers to the representation in which the dynamics is carried out, of which the two most important will be briefly mentioned. In the Molecular Coulomb Hamiltonian $(\mathrm{MCH})$ representation only the kinetic energy of the electrons and coulombic interactions are considered, neglecting external fields and relativistic coupling effects. This is typically the representation in which the electronic structure calculations are performed. Inclusion of the spin-orbit operator renders the Hamiltonian matrix nondiagonal, lifts the degeneracy of states with the same spin multiplicity but different $M_{\mathrm{s}}$, and crucially means the sum of the transition probabilities into all multiplet components is not invariant to molecular rotation in the laboratory frame. To rectify this, the SHARC approach adopts a unitary transform matrix to transform into the so-called diagonal representation,

$$
\mathbf{H}^{\text {diag }}=\mathbf{U}^{\dagger} \mathbf{H}^{\mathrm{MCH}} \mathbf{U},
$$

with all couplings between these diagonal states described by the nonadiabatic coupling matrix $\mathbf{K}^{\text {diag }}$. Such a transformation fulfills the criteria that all couplings are localised and the independence of the sum of the transition probabilities with respect to laboratory frame rotation, and is thus well-suited to surfacehopping simulations of processes involving intersystem crossing.

\section{Computational details}

The differences between the setup parameters of the two sets of simulations (herein labelled simulations $\mathbf{A}$ and $\mathbf{B}$ ) are summarised in Table 1. First, in Section 3.1 below, we discuss the ab initio electronic structure calculations, and second, in Section 3.2, we discuss the simulations.

\subsection{Ab initio electronic structure calculations}

The $a b$ initio electronic structure calculations are carried out using the Molpro software package. ${ }^{54}$ The simulations use the

Table 1 CASSCF active space and number of trajectories included in simulations $\mathbf{A}$ and $\mathbf{B}$. All other parameters were kept identical between simulations. The initial normalised singlet populations at time zero $\left(\mathrm{S}_{0} / \mathrm{S}_{1} / \mathrm{S}_{2} / \mathrm{S}_{3}\right)$ are 0/0.0105/0.8535/0.0915 for simulation $\mathbf{A}$ and 0/0.0176/0.7258/0.0885 for simulation B. Analogously, initial triplet populations $\left(T_{1} / T_{2} / T_{3} / T_{4}\right)$ are 0/0.0249/0.0013/0.0182 and 0/0.0271/0.0025/0.1384 for A and B respectively

\begin{tabular}{lll}
\hline Simulation & A & B \\
\hline Active space & $(8,6)$ & $(10,8)$ \\
Number of trajectories & 571 & 1024
\end{tabular}


turbomole SVP basis set and the state-averaged complete active space self-consistent field (SA-CASSCF) method. SA-CASSCF is a post Hartree-Fock approach that captures most of the static correlation neglected in Hartree-Fock (however not dynamic correlation) via a truncation of the full configuration interaction (CI) set of Slater determinants into a chosen active space of electrons and orbitals, within which all possible permutations are allowed. Orbitals are optimised and each configuration weighted to give the best variational description of the electronic state of interest. In the state-averaged form, the orbitals are optimised to simultaneously describe a manifold of excited states with equal or comparable degree of accuracy. In the current case, the four lowest singlet and triplet states are included in the stateaveraging (SA8).

The orbitals included in the active spaces used in the calculations are shown in Fig. 2. The $(8,6)$ active space in simulation $\mathbf{A}$ contains eight electrons distributed among six orbitals, and expands to a $(10,8)$ active space in simulation B. Both active spaces contain the degenerate sulfur lone pair highest occupied molecular orbitals (HOMOs), the $\sigma$ bonding molecular orbitals (MO) and the $\pi^{*}$ lowest unoccupied molecular orbital (LUMO) pair, labelled $\mathrm{d}-\mathrm{i}$ in Fig. 2. The $(10,8)$ active space features an additional electron pair in the second-highest occupied MO (HOMO-1), and a $\sigma^{*}$ antibonding virtual MO (c and j). In total the $(8,6)$ active space comprises 345 determinants $(225$ singlet, 120 triplet) and $(10,8) 5096$ determinants (3136 singlet, 1960 triplet), illustrating the factorial scaling of computational cost with the size of the active space in CASSCF calculations. It is therefore important to minimise the active space to keep computations tractable, while balancing the quality of the outcomes. Finally, Fig. 2 shows the $(14,10)$ active space used to calculate one-dimensional potential energy curves in radial and angular coordinates using $\operatorname{MRCI}(14,10) /$ aug-cc-pvTZ. While this is too expensive a level of theory on which to run the on-the-fly dynamics, such curves serve as a useful benchmark with which to compare the lower levels of theory.

The calculated adiabatic electronic states can be labeled according to the energy ordering $\left(\mathrm{S}_{0}, \mathrm{~S}_{1}\right.$ etc. for the singlets and $\mathrm{T}_{1}, \mathrm{~T}_{2}$ etc. for the triplets), but also according to the symmetry labels in Table 2, which shows the correspondence between the diabatic and adiabatic state labels at the ground state equilibrium geometry.

\subsection{Simulation parameters and initial condition selection}

The simulations are performed using the surface-hopping code SHARC, ${ }^{48}$ and are combined with electronic structure calculations as already mentioned using the Molpro software package. ${ }^{54}$ To circumvent the severe computational bottleneck imposed by the calculation of full nonadiabatic coupling matrix elements (NACMEs) we turn to wavefunction overlaps, which may be generated by an efficient code integrated in the SHARC package $^{56}$ and offer a faster alternative to full NACME calculation while offering stable numerical propagation of the wavefunction.

For each simulation, trajectories are run for $1 \mathrm{ps}$ with a time step of 0.5 fs. After a surface hop, velocities are rescaled to adjust the kinetic energy in order to conserve the total energy



Fig. 2 Molecular orbitals (MOs) included in the active spaces $(8,6),(10,8)$, and $(14,10)$, corresponding to simulation $\mathbf{A}(\mathbf{d}-\mathrm{i}$, inner rectangle), simulation $\mathbf{B}$ ( $c-j$, center rectangle), and the reference calculations ( $a-j$, outer rectangle). The innermost $(8,6) \mathrm{MOs}(\mathrm{d}-\mathrm{i})$ include the degenerate sulfur lone pair HOMOs, the $\sigma$ bonding MOs and a $\pi^{\star}$ LUMO pair. The $(10,8)$ active space includes a further two MOs (c and j) that correspond to an additional electron pair found in the next-highest occupied $\mathrm{MO}$ and $\mathrm{a} \sigma^{*}$ antibonding virtual $\mathrm{MO}$. Finally, in $(14,10)$ two occupied orbitals $(a$ and $b$ ) are added.

and a decoherence correction is applied. ${ }^{57}$ Initial positions are taken from a Wigner distribution, and initial momenta are assigned to each atom such that the total kinetic energy per molecule approximates the excess kinetic energy from excitation by excitation by a $200 \mathrm{~nm}$ pump pulse to the $S_{2}$ state, using an in-built algorithm in SHARC. In reality such a pulse would access higher-lying electronic states of $\mathrm{CS}_{2}$ that are not included in the current simulations, but here the excess energy serves to ensure that the total energy in the system is sufficient to allow barrier crossing. Initial occupied electronic states are assigned to each trajectory by a probabilistic scheme account for the excitation energies and oscillator strengths at each initial position, again using a built-in algorithm in SHARC originally taken from ref. 58.

In simulation A, 500 initial conditions are generated from the Wigner distribution, from which 573 trajectories are launched 
Table 2 Symmetry labels and correlations for the four lowest-energy singlet and triplet states of $\mathrm{CS}_{2}$ at the linear ground state geometry in the $C_{1}, C_{s}, C_{2 v}$ and $D_{\infty \mathrm{h}}$ point groups (which are used to classify the electronic states). The $C_{1}$ point group has no symmetry and simply corresponds to the energy ordering of the adiabatic singlet and triplet states. Assignments in the $D_{\infty \mathrm{h}}$ point group are taken from ref. 55

\begin{tabular}{lllll}
\hline Point group & $C_{1}$ & $C_{\mathrm{s}}$ & $C_{2 \mathrm{v}}$ & $D_{\infty \mathrm{h}}$ \\
\hline State/notation & $\mathrm{S}_{0}$ & ${ }^{1} \mathrm{~A}^{\prime}$ & ${ }^{1} \mathrm{~A}_{1}$ & ${ }^{1} \Sigma_{\mathrm{g}}^{+}$ \\
& $\mathrm{S}_{1}$ & ${ }^{1} \mathrm{~A}^{\prime \prime}$ & ${ }^{1} \mathrm{~A}_{2}$ & ${ }^{1} \Sigma_{\mathrm{u}}^{-}$ \\
& $\mathrm{S}_{2}$ & ${ }^{1} \mathrm{~A}^{\prime}$ & ${ }^{1} \mathrm{~B}_{2}$ & ${ }^{1} \Delta_{\mathrm{u}}$ \\
& $\mathrm{S}_{3}$ & ${ }^{1} \mathrm{~A}^{\prime \prime}$ & ${ }^{1} \mathrm{~A}_{2}$ & ${ }^{1} \Delta_{\mathrm{u}}$ \\
& $\mathrm{T}_{1}$ & ${ }^{3} \mathrm{~A}^{\prime}$ & ${ }^{3} \mathrm{~B}_{2}$ & ${ }^{3} \Sigma_{\mathrm{u}}^{+}$ \\
& $\mathrm{T}_{2}$ & ${ }^{3} \mathrm{~A}^{\prime \prime}$ & ${ }^{3} \mathrm{~A}_{2}$ & ${ }^{3} \Delta_{\mathrm{u}}$ \\
& $\mathrm{T}_{3}$ & ${ }^{3} \mathrm{~A}^{\prime}$ & ${ }^{3} \mathrm{~B}_{2}$ & ${ }^{3} \Delta_{\mathrm{u}}$ \\
& $\mathrm{T}_{4}$ & ${ }^{3} \mathrm{~A}^{\prime \prime}$ & ${ }^{3} \mathrm{~A}_{2}$ & ${ }^{3} \Sigma_{\mathrm{u}}^{-}$
\end{tabular}

(an initial condition may be used to launch trajectories on more than one state due to the probabilistic selection of initial states). Trajectories that fail to reach $t_{\max }=1 \mathrm{ps}$, for instance due to convergence problems of the CASSCF electronic structure calculations at a particular time step, are treated in the following manner. If prematurely-terminated trajectories have dissociated before the point of failure, the dissociating sulfur atom is propagated to $t_{\max }$ at the average velocity between the point of dissociation and the last successful time step, with the last recorded $\mathrm{MCH}$ state taken as final (since surface hops within a particular spin multiplicity beyond dissociation have no meaningful effect on the branching ratio). Trajectories that have not dissociated before failure are discarded. By this procedure, 571 successful trajectories were obtained.

In simulation $\mathbf{B}$, a larger set of 1000 initial conditions are generated from the Wigner distribution to compensate for more frequent CASSCF failures due to the larger active space. A restart procedure is applied to trajectories which fail to reach 1 ps as follows:

(1) Re-running the point-of-failure time step with internal orbital optimisation in the CASSCF step turned off, and turning it back on if the step is successful.

(2) Failing that, if the trajectory is dissociative (defined as one bond being $3 \AA$ or longer), that bond is extended by a small percentage $(1 \%, 2 \%$ or $5 \%$ depending on the severity of the case) and continued from the new coordinates.

Following these steps, the same assumptions in terms of propagation of the dissociated sulfur atom and its $\mathrm{MCH}$ state as in simulation $\mathbf{A}$ are applied. Since the restart procedure inevitably reduces the quality of the trajectory, the whole dataset was scanned for trajectories exhibiting discontinuous behaviour, such as unphysically large jumps in bond length during a single time step (seen most prevalently where step 2 of the above procedure was applied to trajectories with already large $\mathrm{C}-\mathrm{S}$ distances). Such trajectories, comprising approximately $8 \%$ of the bunch, were filtered out, resulting in no qualitative change in the final results. Because the restart procedure applied only to trajectories past the dissociation barrier, it had no effect on the final singlet/triplet branching ratio or pre-barrier dynamics. These procedures gave a total of 1024 included trajectories for simulation $\mathbf{B}$.

\section{Results and discussion}

\subsection{Ab initio calculations}

To get an idea of the performance of each level of theory, it is instructive to examine one-dimensional potential energy cuts along coordinates of interest, as shown in Fig. 3 and 4 . What is immediately obvious is that despite the relatively simple premise of photodissociation in a small triatomic molecule, the underlying potential energy landscape is complex with many degeneracies, near-degeneracies, conical intersections and Renner-Teller splittings.

Fig. 3 shows the potentials along the $\Theta_{\mathrm{SCS}}$ bending coordinate. Both the $(8,6)$ and $(10,8)$ levels of theory replicate the angular potentials of the benchmark $\operatorname{MRCI}(14,10)$ calculations rather well, with the predicted vertical $S_{2} \leftarrow S_{0}$ excitation energy $4.16 \mathrm{eV}$ for $(8,6), 4.29 \mathrm{eV}$ for $(10,8)$, and $4.12 \mathrm{eV}$ for $\operatorname{MRCI}(14,10)$ (see also Table 3). From the closely-packed nature of the states in the excitation region, one would expect rapid redistribution of the excited population via nonadiabatic and spin-orbit coupling already in the early stages of the dynamics. Combined with the high kinetic energy enforced on the system $(2.5 \mathrm{eV}$, to make the barrier clearly accessible) and the tendency of the excited states to have their minimum energy at bent geometry, one would hazard to predict strong bending motion to be observed

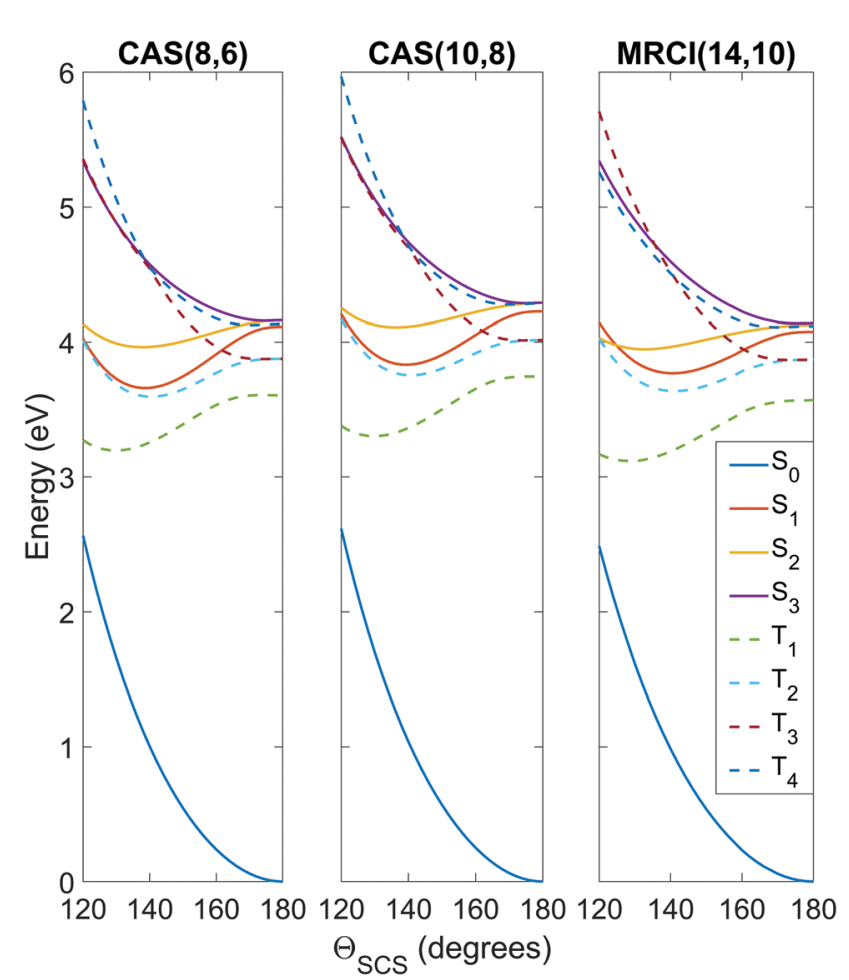

Fig. 3 Potential energy curves as a function of the $\Theta_{\text {SCS }}$ bending coordinate, calculated at the SA8-CAS(8,6)/SVP (simulation A), SA8-CAS $(10,8) / S V P$ (simulation B), and $\mathrm{MRCI}(14,10)$ /aug-cc-pvTZ (reference) levels of theory, shown in the left, center and right panels. Bond lengths are fixed at the CASSCF $(16,12) /$ aug-cc-pvQZ optimised value of $1.569 \AA$. For compactness, only the range $120^{\circ} \leq \Theta_{\mathrm{SCS}} \leq 180^{\circ}$ is shown as the curves are symmetric about the linear geometry at $\Theta_{\mathrm{SCS}}=180^{\circ}$. Here the states are labelled adiabatically. A full conversion table of adiabatic and diabatic state labels is given in Table 2. 

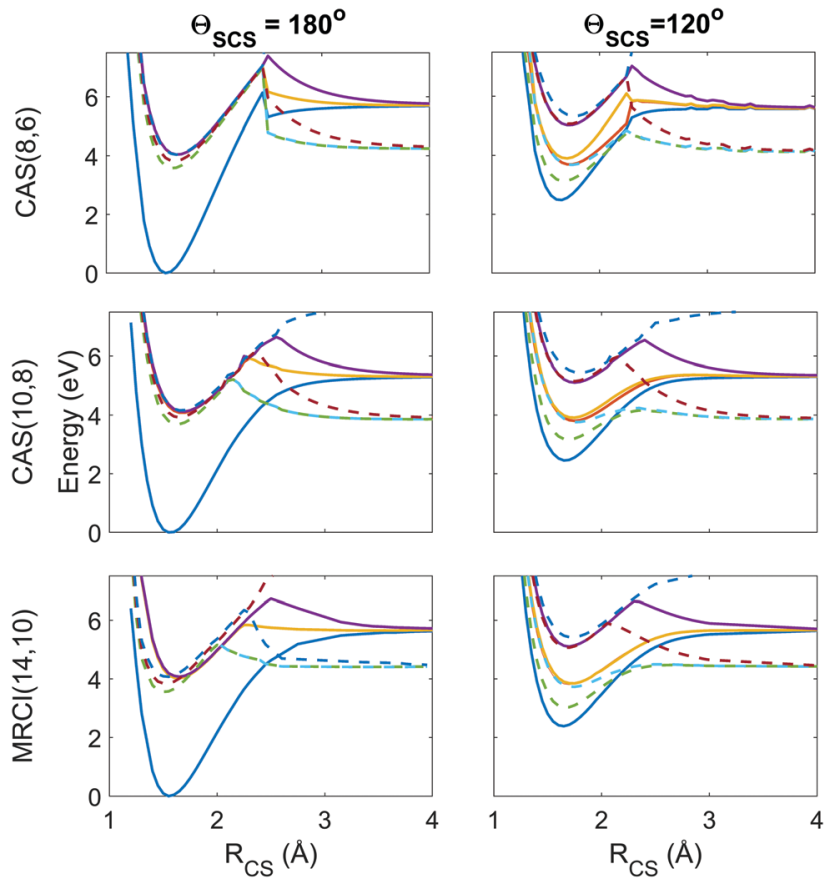

$$
-\mathrm{S}_{0}-\mathrm{S}_{1}-\mathrm{S}_{2}-\mathrm{S}_{3}--\mathrm{T}_{1}--\mathrm{T}_{2}--\mathrm{T}_{3}--\mathrm{T}_{4}
$$

Fig. 4 Potential energy curves as a function of the $R_{\mathrm{CS}}$ stretch coordinate, with the second bond length fixed at $R_{\mathrm{CS}}=1.569 \AA$ and the angle $\Theta_{\mathrm{SCS}}$ fixed at linear $180^{\circ}$ (left column) and bent $120^{\circ}$ (right column), with calculations at the SA8-CAS $(8,6) / S V P$ (simulation A), SA8-CAS $(10,8) / S V P$ (simulation B), and $\mathrm{MRCI}(14,10) /$ aug-cc-pvTZ (reference) level shown in the top, middle and bottom rows. Here the states are labelled adiabatically. A full conversion table of adiabatic and diabatic state labels is given in Table 2 .

in the simulations. In this regard, the stories presented by the $(8,6)$ and $(10,8)$ levels of theory used in simulations $\mathbf{A}$ and $\mathbf{B}$ are consistent, and their angular potentials show comparatively small qualitative differences.

Fig. 4 shows the radial potential energy curves along the $R_{\mathrm{CS}}$ stretch coordinate corresponding to dissociation of one sulfur. In contrast to the angular curves, the active space must describe accurately not just the reactant but also the products while accounting for the electron correlation during bond breaking. The deficiencies in the $(8,6)$ active space used in simulation $\mathbf{A}$ begin to manifest as discontinuities and severe exaggerations of the barrier height towards dissociation. This is particularly prevalent in the highest state considered, $\mathrm{T}_{4}$, however this state is energetically inaccessible during the simulations. Also noteworthy are unphysical undulations in the potential energy curves beyond the barrier in the smaller $(8,6)$ calculations at $\Theta_{\text {SCS }}=120^{\circ}$, but these have negligible effect on the predissociation dynamics. The potential wells for the $(8,6)$ calculations have sharper gradients along the stretch coordinate than in $(10,8)$, so one may expect the vibrational motion to be faster in simulation $\mathbf{A}$. Overall, the radial potential energy curves are much smoother in the $(10,8)$ calculations (i.e. simulation $\mathbf{B})$, with no discontinuities and a closer match to the $\operatorname{MRCI}(14,10)$ reference potentials. This suggests the inclusion of the antibonding occupied and virtual orbitals (c) and (j) in Fig. 2 is crucial to a proper description of
Table 3 Predicted oscillator strengths $f_{i j}$ at bent geometry with $\Theta_{\mathrm{SCS}}=$ $170^{\circ}$ and excitation energies $\Delta E$ at linear geometry with $\Theta_{\mathrm{SCS}}=180^{\circ}$ for the three lowest excited singlet states, calculated at SA8-CAS $(8,6) / \mathrm{SVP}$ (simulation A), SA8-CAS(10,8)/SVP (simulation B) and MRCI(14,10)/aug-ccpvTZ (reference) level of theory (taken from ref. 10). In all cases the molecule has the equilibrium bond length $R_{\mathrm{CS}}=1.569 \AA$



bond breaking in this system; test calculations show that the extra electron pair is primarily responsible for lowering the barrier height while the extra virtual orbital serves to smooth out the potentials in the barrier region. This effect is further observed in the recent work of Trabelsi et al. ${ }^{62}$ where higherlevel MRCI calculations yield even smoother potential energy curves. For a more general discussion on active space selection in CASSCF problems, the reader is directed to ref. 59-61 and citations therein.

The importance of spin-orbit coupling in this system is emphasised by the strong mixing between individual components of different triplet manifolds at equilibrium geometry $e . g$. the 5 th spin-coupled state (corresponding to one of the states in the degeneracy between $\mathrm{T}_{2}$ and $\mathrm{T}_{3}$ ) consisting of $\approx 50 \%$ each of the $\mathrm{T}_{2}^{-}$and $\mathrm{T}_{3}^{+}$multiplet components, or the 16th spin-free state comprising $98.4 \%$ of $\mathrm{S}_{3}$ and the remainder a non-negligible contribution from $\mathrm{T}_{2}^{0}$. Such effects are extremely dependent on geometry but feature markedly in the dynamics.

Predicted oscillator strengths at each level of theory at $\Theta_{\text {SCS }}=170^{\circ}$ are shown in Table 3 together with excitation energies. These off-linear appearances of non-zero oscillator strengths for transitions formally forbidden are a well-known effect, accounted for here by Wigner initial conditions sampling.

\subsection{Simulations (population dynamics)}

We now turn to the simulations, and begin by considering the populations on the electronic states as a function of time. The total populations on all singlet and triplet states are shown in Fig. 5, with the data taken from simulations A and B. Here, the populations are defined as the normalised summed squares of the MCH coefficients of each state. In both simulations there is a rapid decay in the singlet population (and commensurate rise in the triplet) for $t>0$, which is greater in simulation A. By the end of the simulations at $1 \mathrm{ps}$, the singlet/triplet fraction for simulation $\mathbf{A}$ is $0.25 / 0.75$ compared to $0.32 / 0.68$ in simulation $\mathbf{B}$, although the curves have not quite reached a plateau by 1 ps.

We continue towards a more mechanistic picture of the dynamics where the singlet and triplet populations are categorised as bound or dissociated, as shown in Fig. 6. The populations are calculated classically, i.e. using a straightforward normalised sum of the number of trajectories occupying singlet or triplet $\mathrm{MCH}$ states, with dissociation defined as one of the CS distances being longer than 2.73 or $2.96 \AA$ in simulation A or B, respectively. The thresholds are defined as the minimum CS distance at which 


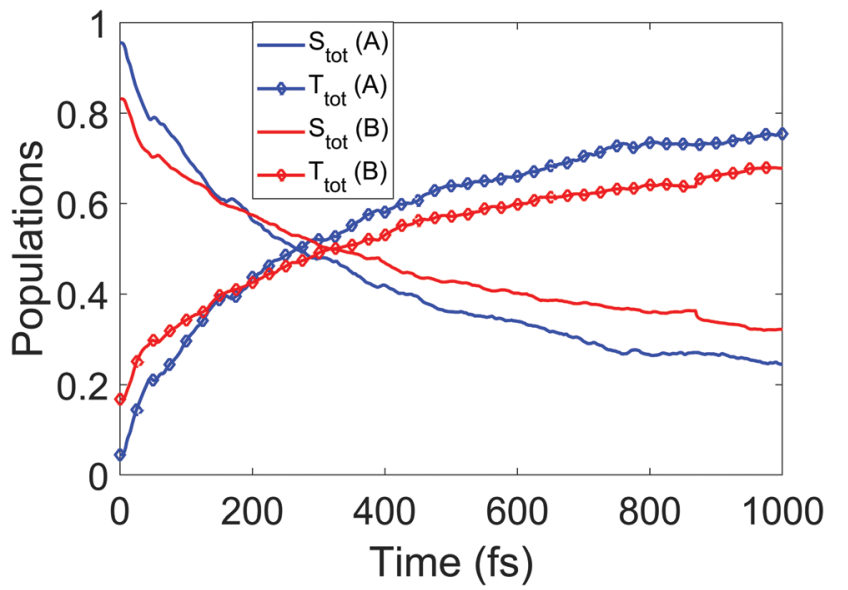

Fig. 5 Total singlet and triplet populations (quantum) as a function of time, defined as the sum of the squares the $\mathrm{MCH}$ state coefficients, for simulations $\mathbf{A}$ and $\mathbf{B}$.

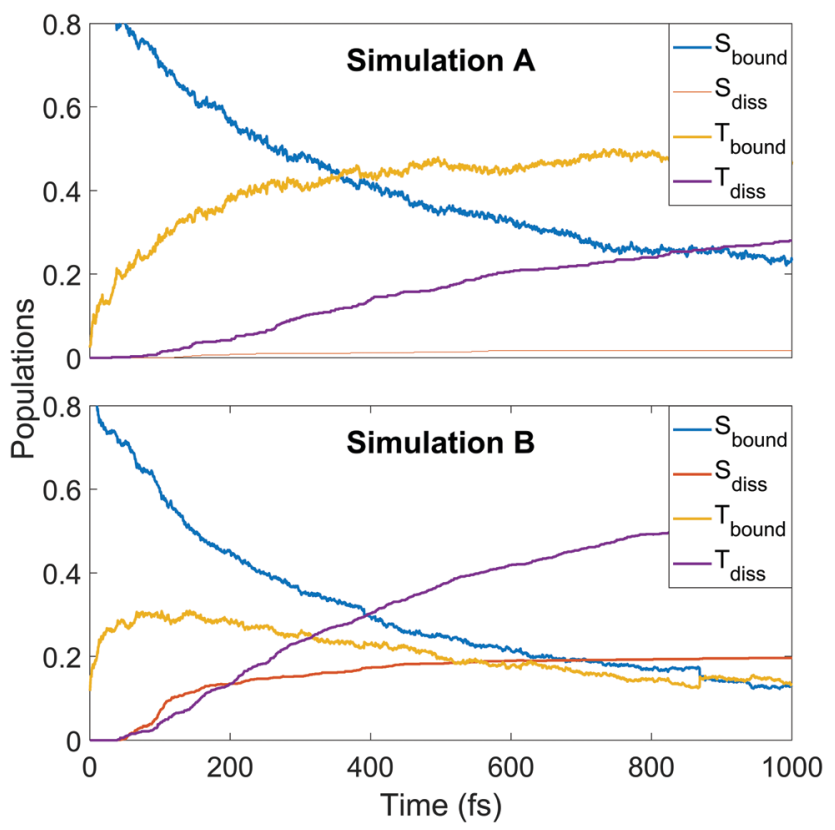

Fig. 6 Populations of singlet and triplet states in simulation A (upper panel) and $\mathbf{B}$ (lower panel), separated into contributions from bound and dissociated trajectories.

dissociation is irreversible in each simulation. The decay of the bound singlet population follows a similar profile in both simulations, decaying exponentially to approximately $15-20 \%$ of the total population by $1 \mathrm{ps}$. The other curves are qualitatively similar but differ in magnitude. The initial transfer into bound triplet at very early times is similar in both simulations, but is followed by a further rise before maintaining steadily near $50 \%$ in simulation $\mathbf{A}$. This is in contrast to simulation $\mathbf{B}$, where the initial rise is followed by a steady decay into the dissociation channels. Both dissociation channels are slower to rise in $\mathbf{A}$, with the triplet channel activating well before the singlet. The opposite is true in $\mathbf{B}$, where both channels activate at $50 \mathrm{fs}$ into the dynamics with the singlet channel initially dominating, before shutting off while the triplet channel continues to rise until the end of the simulation. This latter pattern of behaviour is seen qualitatively in $\mathbf{A}$, but the dissociation fractions are lower.

Thus the conclusions drawn in Section 4.1 from the simple one-dimensional potential energy cuts earlier are borne out, in that the higher barriers and steeper potentials in simulation $\mathbf{A}$ lead to a trapping of population in the triplet states (mediated by the spin-orbit coupling) and frustrated dissociation. The difference in the potential energy landscape have significant effect on the final reported dissociation fractions $(1.8 \%$ singlet and $28 \%$ triplet in A, and $20 \%$ singlet and $54 \%$ triplet in B as fractions of the total trajectory ensembles) and corresponding ratios (singlet:triplet ratio $1: 15.6$ for $\mathbf{A}$ and $1: 2.8$ for $\mathbf{B}$ ). Therefore the exaggerated barriers to dissociation in $\mathbf{A}$, while obviously impacting the overall dissociated population, also emphasise the easier access to the triplet channel, which accounts for a far higher proportion of the dissociated population in $\mathbf{A}$ with respect to $\mathbf{B}$, where the singlet dissociation barrier is even harder to overcome.

We finish the discussion of the populations with a detailed view of the state-by-state populations as shown in Fig. 7. Most of the initial population in both simulations is naturally found in the bright $\mathrm{S}_{2}$ state. There is an extremely rapid distribution of population from this state (whose population drops to below $50 \%$ in less than $50 \mathrm{fs}$ in both cases) amongst the other singlet states, mainly into $S_{3}$ and $S_{1}$. After the initial redistribution, $\mathrm{S}_{3}$ quickly loses its accrued population while $\mathrm{S}_{1}$ continues a slight rise until $\approx 200$ fs. From this point, $\mathrm{S}_{1-3}$ all decay steadily. The ground state population is slower to rise in $\mathbf{A}$, taking around $90 \mathrm{fs}$ before remaining steady for the rest of the dynamics at about $7 \%$. In $\mathbf{B}$, there is an initial rise in $\mathrm{S}_{0}$ after $30 \mathrm{fs}$, a short plateau, and a second rise before leveling out at about $9 \%$. It should be noted that after approximately $500 \mathrm{fs}$ in both cases, major activity in the singlet states has ceased. This is most pronounced in $\mathbf{A}$, while in $\mathbf{B}$ some very slow decay in $S_{1}$ and $S_{2}$ remains even at later times.

Some distinct fluctuations are visible in the populations, most notably an increase in $\mathrm{S}_{2}$ population in the interval 40 to $75 \mathrm{fs}$. Analysis of the surface hops in and out of $\mathrm{S}_{2}$ in this period reveal that the net flux gain is due to an influx of population from $\mathrm{S}_{3}$ and a relatively minor contribution from $\mathrm{T}_{3}(93 \%$ and $7 \%$ of net flux in $\mathbf{B}$, where flux is simply defined as the difference between the number of hops into and hops out of $\mathrm{S}_{2}$ ). Looking at the state-resolved triplet populations one sees that these states also play a strong role in the early redistribution of population, reinforcing the importance of spin-orbit coupling in the early-time dynamics of the system. Other features that emerge in the first $100 \mathrm{fs}$ are early out-of-phase oscillations between $\mathrm{T}_{3}$ and $\mathrm{T}_{4}$, the secondary role of $\mathrm{T}_{3}$ (whose barrier to dissociation tends to lie higher in energy than that of $\mathrm{T}_{1}$ and $\mathrm{T}_{2}$ ) at later times, the near-commensurate rise in $\mathrm{T}_{1}$ and $\mathrm{T}_{2}$ (clearer in simulation $\mathbf{B}$ ) reflecting the closely-spaced nature of the respective potential energy surfaces of those states, and the steady hold of population in $\mathrm{T}_{4}$, whose high barrier to dissociation allows this state to act as a reservoir before the stored population eventually is transferred elsewhere. Analysis of the origin of hops into $\mathrm{T}_{3}$ over the first 50 fs shows that the largest contributions come from $S_{3}$ and $T_{4}$ 



Fig. 7 State-resolved populations as a function of time for each of the simulations. The rows refer to simulation $\mathbf{A}$ and $\mathbf{B}$ (upper and lower respectively) and the columns to singlet and triplet states (left and right respectively).

(55\% and $37 \%$ of net flux respectively in simulation B). The hopping analysis explains why $S_{3}$, after gaining so much population in the initial redistribution via internal conversion, decays as rapidly as it does: first to $\mathrm{T}_{3}$ until $\approx 50 \mathrm{fs}$, then back to $\mathrm{S}_{2}$ up to $\approx 75 \mathrm{fs}$. This analysis shows clearly the direct competition between internal conversion and intersystem crossing characteristic of this molecule.

\subsection{Simulations (structural dynamics)}

We now turn to the structural dynamics. Shown in Fig. 8 are the average CS bond lengths separated into contributions from bound and dissociated trajectories, and the average $\Theta_{\text {SCS }}$ angle for the bound trajectories. Again, similar behaviours are seen in both simulations $\mathbf{A}$ and $\mathbf{B}$. In terms of the bond lengths, it is clear that initially it is the symmetric stretch that is excited, and it takes around $300 \mathrm{fs}$ before these clear oscillations disperse. The impact of the higher dissociation barrier on the predicted dynamics in simulation A can be seen by the two clear oscillations seen in the bound $R_{\mathrm{CS}}$ curve, compared to only one in simulation $\mathbf{B}$, reflecting the difficulty trajectories have in getting over the barrier in A. A similar effect is seen in the average angle in that it is slower to damp in $\mathbf{A}$ than $\mathbf{B}$.

In Fig. 9, snapshots of the nuclear densities associated with the sulfur atoms are shown at a series of time points $(0,50,100$ and $1000 \mathrm{fs}$ ) for each simulation. These are calculated by projecting the internal coordinates onto the $(x, y>0)$ plane with the carbon atom placed at the origin. Only the probability density associated with the two sulfur atoms is shown, and this is calculated at each point in the plane as,

$$
P(x, y)=\left(N_{\text {traj }}\right)^{-1} \sum_{i=1}^{N_{\text {traj }}} \sum_{j=1}^{N_{\mathrm{S}}}\left(\frac{\gamma}{\pi}\right) \exp \left(-\gamma\left[(x, y) \vec{Q}_{i j}\right]^{2}\right),
$$

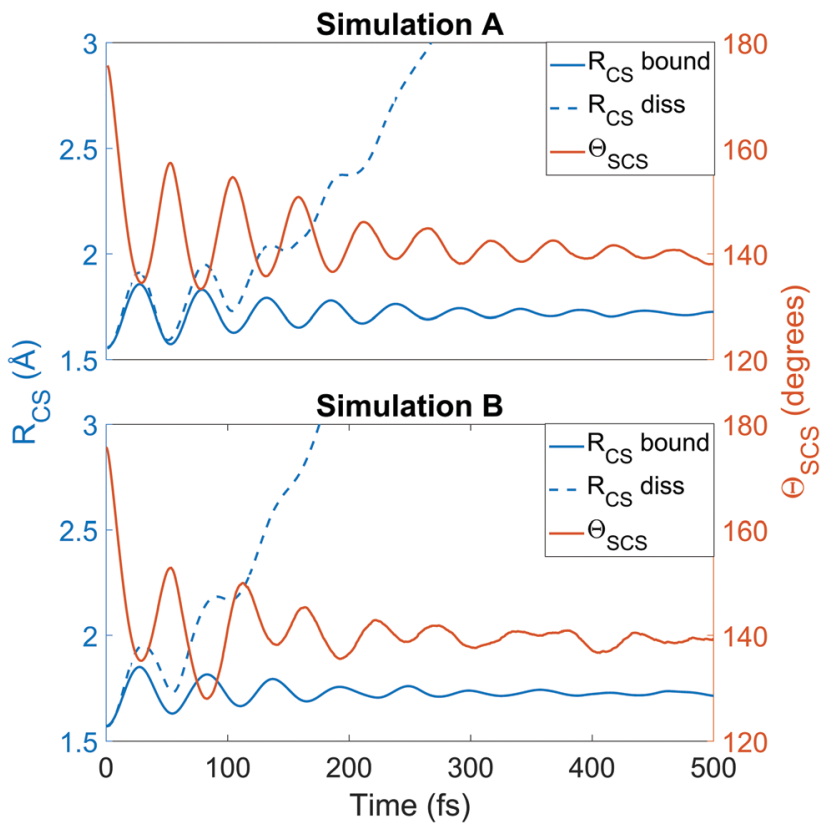

Fig. 8 Average $R_{\mathrm{CS}}$ for bound and dissociated molecules and average $\Theta_{\mathrm{SCS}}$ for bound molecules, calculated for simulation $\mathbf{A}$ (upper) and $\mathbf{B}$ (lower) as a function of time. The average bound geometry is calculated for all molecules up until they dissociate (i.e. CS fragments are excluded). The average $\Theta_{\text {SCS }}$ is calculated from all trajectories as long as they remain intact (not dissociated). A trajectory is designated as dissociated when one $R_{C S}$ exceeds the minimum distance from which dissociation is irreversible in each simulation.

where $\vec{Q}_{i j}$ are the coordinates of sulfur atom $j$ in trajectory $i$ in the plane, $\gamma$ is a Gaussian width parameter equal to $1 /\left(2 \sigma^{2}\right)$ where $\sigma=0.05, N_{\mathrm{S}}$ is the number of sulfur atoms and $N_{\text {traj }}$ is the 

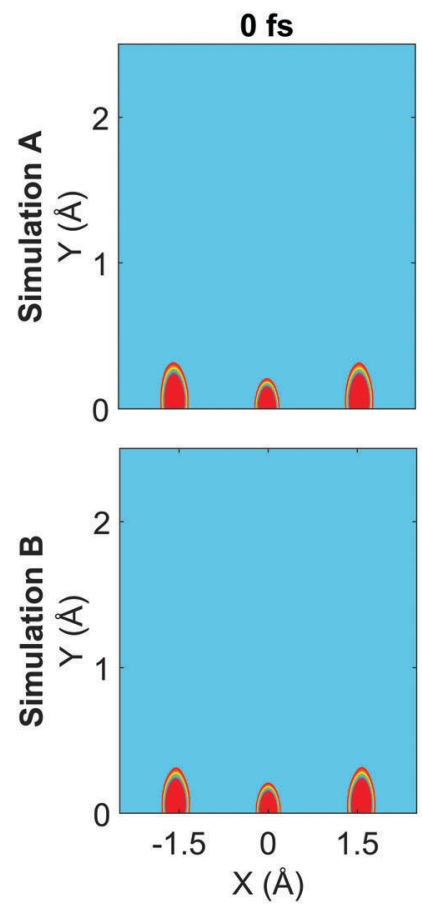

$50 \mathrm{fs}$
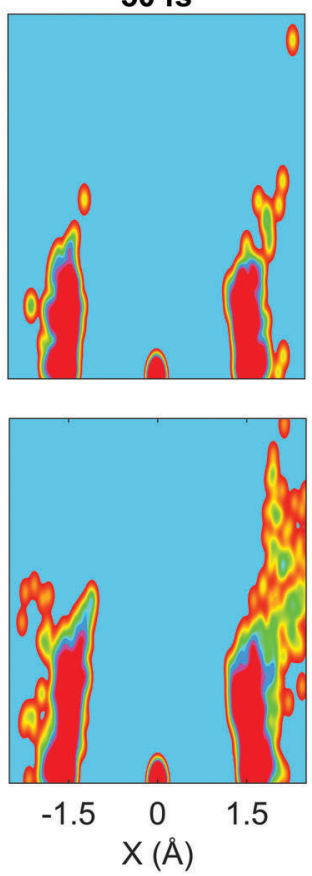

$100 \mathrm{fs}$
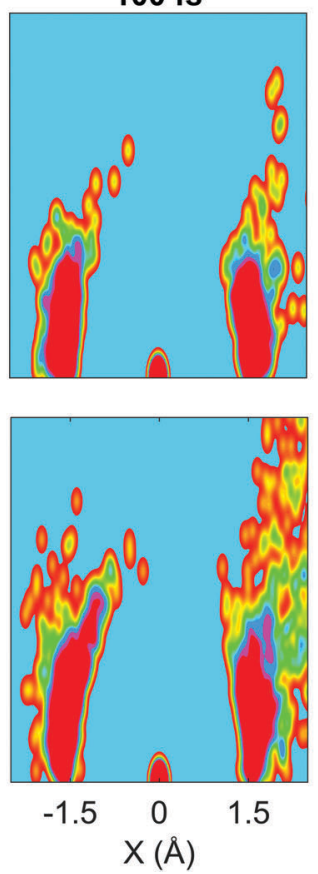

$1000 \mathrm{fs}$
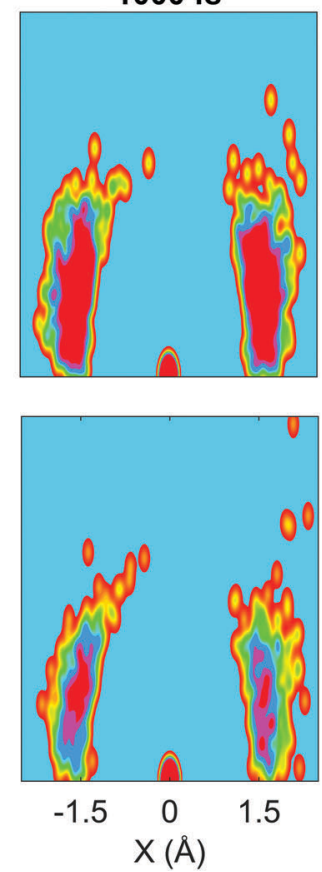



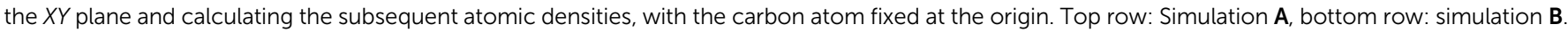
Full movies for each simulation available in ESI. $\dagger$

number of trajectories. The probability density $P(x, y)$ thus amounts to a convolution of the classical coordinates of the sulfur atoms with a normalised Gaussian function. This manner of plotting the evolution of the molecular structure provides a simple way of visualising nuclear motion and its dispersion as the reaction unfolds, while separating out electronic effects. In both simulations, at time zero all atoms are nicely localised around the equilibrium geometry, taking into account the spread of the Wigner distribution of initial positions. By $50 \mathrm{fs}$ the dissociation pathway is clearly manifesting, notably to a greater extent in simulation $\mathbf{B}$ (albeit that the shape of the nondissociated part of the wavepacket is approximately the same in both simulations). The spread of the vibrational wavepacket is rapid, with $\Theta_{\text {SCS }}$ ranging from $180^{\circ}$ to $120^{\circ}$. This theme continues in the two remaining snapshots, with the extent of dissociation clearly growing at 100 fs before greatly reducing by the end of the simulations at $1000 \mathrm{fs}$ (reflecting the evolution of populations in Fig. 6). In simulation A, the dissociative pathway has completely stalled; this is not the case in simulation $\mathbf{B}$ where the dissociation of the sulfur atom is clearly still active. Full movies constructed from such snapshots are available as Movie $\mathrm{CS}_{2} \mathbf{A}$ and Movie $\mathrm{CS}_{2}$ $\mathbf{B}$ in the ESI. $\dagger$

Fig. 10 collates the molecular geometry and time-point for each surface hop in the higher-level simulation B. Each data point represents an individual surface hop, defined by the two CS bond lengths, the bending angle, and the time of occurrence. The three panels show all hops within singlet states, triplet states, and between singlet and triplet states. The clusters provide insight into the correlation between nonadiabatic and spin-orbit coupling effects and the molecular geometry, as well as the evolution of the dynamics. Common to each subfigure is a dense cluster centered around early times at near equilibrium geometry, representative of the rapid internal conversion and intersystem crossing at early times and the concurrent competition between these two distinct processes. This feature is naturally less pronounced in the plot of triplet-triplet hops only, as nonadiabatic hops within the triplet manifold must be preceded by a intersystem crossing into that manifold (barring the small number of trajectories that originate in a triplet state). There is an additional well-defined cluster in the singlet-to-singlet panel corresponding to one elongated CS bond and a narrow distribution of acute bending angles, reflective of the other main region the different singlet electronic states come closer together in energy as they approach and exceed the barrier crossing region. This is seen to be broadly symmetric across both bonds. A similar effect is seen in the tripletto-triplet panel, but here it is not nearly as restricted in terms of angular range due to the smaller variation of energy spacing with bending angle in the triplet states. The spin-orbit coupling (intersystem crossing) surface hops in the singlet-triplet panel are in contrast are far more tightly clustered in the Franck-Condon region because trajectories spending time is this region are constantly exposed to singlet and triplet states lying close to each other.

\subsection{Branching ratios, convergence, and timings}

The final branching ratio between singlet and triplet dissociation is given in Table 4. Both simulations show the same qualitative trend, in that the triplet pathway is the dominant dissociation 


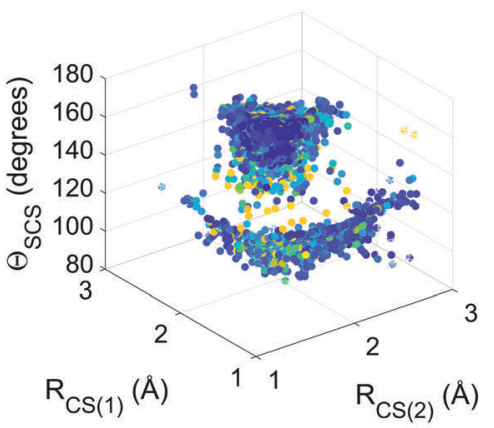

(a) Singlet-singlet transitions (IC)

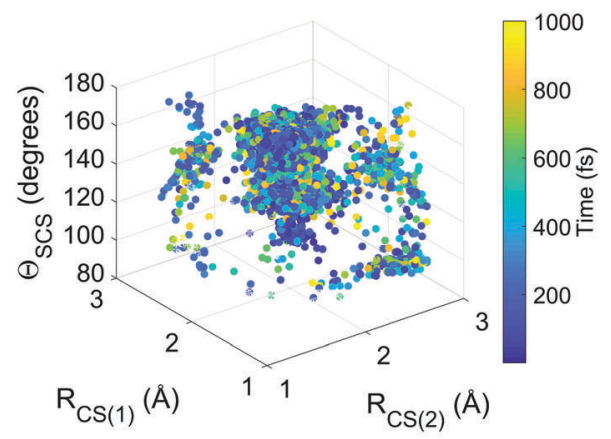

(b) Triplet-triplet transitions (IC)



(c) Singlet-triplet transitions (ISC)

Fig. 10 Four-dimensional plots of surface hops. Each point represents a hop between electronic states, with the molecular geometry represented by its position and the time by its colour. Hops are grouped by multiplicity. Singlet-singlet and triplet-triplet hops correspond to nonadiabatic transitions, i.e. internal conversion (IC), while singlet-triplet hops correspond to spin-orbit coupling, i.e. intersystem crossing (ISC).

Table 4 Branching ratio of singlet to triplet state dissociated sulfur atoms at the end of the simulation at 1 ps, for simulations $\mathbf{A}$ and $\mathbf{B}$

\begin{tabular}{lll}
\hline Simulation & S branch & T branch \\
\hline A & 0.059 & 0.941 \\
B & 0.267 & 0.733
\end{tabular}

pathway due to its lower barrier to dissociation supported by the strength of spin-orbit coupling in the system. This ratio is exaggerated in simulation $\mathbf{A}$, as a consequence of the overestimation of the barriers to dissociation in the singlet states. We note that although a pathway that results in $\mathrm{C}+\mathrm{S}_{2}$ fragments is possible, ${ }^{62}$ this dissociation channel is not observed in our simulations.

To measure convergence of each simulation, we use two measures; the branching ratio as a function of the number of dissociated trajectories, and the state populations at $t=1 \mathrm{ps}$ as a function of all trajectories. For each metric, we calculate the variance $V(N)$ (or the mean of all the $V(N)$ in the case of final state populations) for random subsets of trajectories for $N \in\left[1, N_{\text {traj }}\right]$, with,

$$
V(N)=\sqrt{(B(N)-\langle B\rangle)^{2}}
$$

where $\langle B\rangle$ and $B(N)$ are the final predicted branching ratio/state populations and the branching ratio/state populations of a subset consisting of $N$ trajectories respectively. The variance calculation is repeated 1000 times for each $N$ with the $N$ trajectories chosen at random each time, and the result is averaged. This procedure generates the plots shown in Fig. 11. For the branching ratio, convergence is faster in simulation $\mathbf{A}$ with the variance halving from its initial value in only $\approx 10$ trajectories. Convergence is much smoother in simulation $\mathbf{B}$, decreasing rapidly in a similar number of trajectories as the first simulation. A similar trend is seen in terms of population convergence with simulation $\mathbf{A}$ converging in fewer trajectories than in simulation B. In this case both curves decrease smoothly.

Example timing information between the two simulations is shown in Table 5. For simplicity, these are based on four representative trajectories with identical initial conditions, run with either the $(8,6)$ or $(10,8)$ active spaces and either the overlap or NACME coupling schemes. Whilst merely illustrative, these give approximate measures as to the growth in expense from method to method. Changing the coupling scheme from overlaps to NACMEs increases per-time step expense by $78 \%$ and $119 \%$ for the $(8,6)$ and $(10,8)$ active spaces respectively, while increasing the active space from $(8,6)$ to $(10,8)$ increases expense by $337 \%$ and $434 \%$ for overlap and NACME simulations respectively.

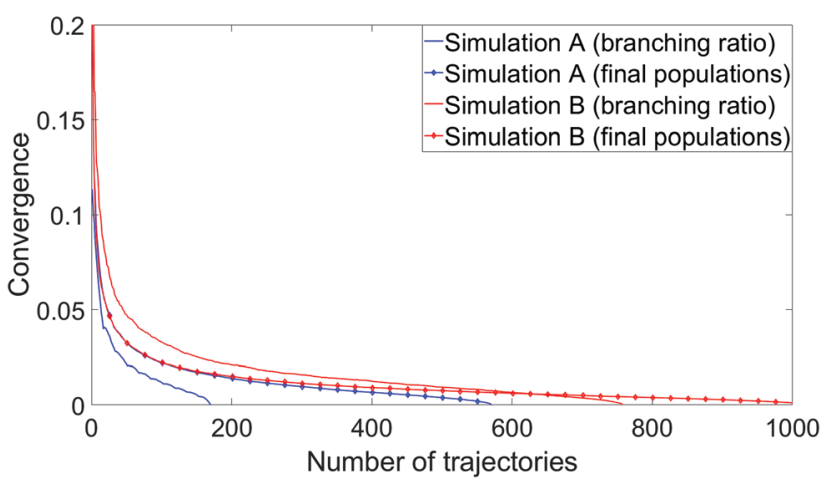

Fig. 11 Convergence plots of each simulation as a function of the branching ratio and the final predicted state populations. In the former case, convergence is defined as the variance from the final predicted branching ratio of a random subset of dissociated trajectories as a function of the number of trajectories selected. In the latter case, the convergence metric is the mean of the variances of all state populations at $t=1 \mathrm{ps}$. In each case the random selection and variance calculation was repeated 1000 times and the results averaged. 
Table 5 Example timing information that illustrates the difference in computational expense for the different simulations. These are based on the reported per-time step computation times of four trajectories whose initial conditions are identical, run for $100 \mathrm{fs}$ in $0.5 \mathrm{fs}$ steps with either the $(8,6)$ or $(10,8)$ active space and with either the overlap or nonadiabatic coupling matrix elements (NACMEs) coupling schemes. These were run on independent compute nodes at the ECDF HPC (64 GB RAM, Intel ${ }^{\mathbb{R}}$ Xeon ${ }^{\mathbb{R}}$ processor E5-2630 v3, 2.4 GHz). The quoted total ab initio time accounts for the fact that each substep requires multiple integral, gradient and NACME computations

\begin{tabular}{lllll}
\hline Wall clock time (s) & $\begin{array}{l}(8,6) / \\
\text { overlap }\end{array}$ & $\begin{array}{l}(8,6) / \\
\text { NACME }\end{array}$ & $\begin{array}{l}(10,8) / \\
\text { overlap }\end{array}$ & $\begin{array}{l}(10,8) / \\
\text { NACME }\end{array}$ \\
\hline Integrals & 0.696 & 0.701 & 0.749 & 0.813 \\
CASSCF & 1.620 & $1.620^{a}$ & 3.942 & $3.942^{a}$ \\
Gradients & 2.689 & 2.476 & 14.037 & 11.905 \\
Spin-orbit coupling & 3.379 & 3.347 & 7.449 & 7.831 \\
NACMEs & - & 2.318 & - & 14.365 \\
WF overlaps & 1.017 & - & 1.152 & - \\
Total $a b$ initio time & 28.970 & 51.791 & 126.470 & 276.625
\end{tabular}

${ }^{a}$ In NACME simulations no separate CASSCF-only calculation is carried out, but this value can be approximated by the corresponding overlap simulation.

Accounting for the fact that multiple NACMEs and gradients are calculated at each step, it is those components which contribute the most to total compute time.

\section{Conclusions}

We have compared two different simulations of the photodissociation of $\mathrm{CS}_{2}$ when the molecule is excited to the first ${ }^{1} \mathrm{~B}_{2}$ state. The simulations consist of swarms of surface-hopping trajectories evolving on potential energy surfaces generated on-the-fly as implemented in SHARC, ${ }^{48}$ with electronic structure calculations at the SA-CASSCF level in Molpro. ${ }^{54}$ The chief difference between the simulations lies in the choice of active space, $(8,6)$ versus $(10,8)$, and we examine the impact this has on the dynamical outcomes. The smaller active space is shown to exhibit frustrated dissociation primarily due to the excessively high potential barriers wrought by the more limited active space, whose orbitals fail to describe the bond-breaking regime of the potential energy landscape adequately. This deficiency is not observed in the larger active space, where the addition of only two extra orbitals (one occupied, one virtual) greatly enhances the description of the chemistry. Naturally, further improvements could be made with an even larger active space, as exemplified by the reference $a b$ initio potential energy curves calculated at the MRCI $(14,10) /$ aug-cc-pvTZ level. However, the computational cost would be extreme if the goal was to include both nonadiabatic and spin-orbit coupling in the on-the-fly dynamics at this level.

An important observation is that examination of potential energy curves along key coordinates provides a reasonably reliable prediction of the nature and shortcomings of the dynamics in systems whose potential energy landscape contains distinct topological features such as dissociation barriers, especially if more accurate potential energy curves are available for reference.
Therefore, it is often valuable to include such representative potential energy curves (which may correspond to minimum energy paths for systems of high dimensionality where the reaction coordinate is not obvious) alongside published simulations, preferably accompanied by accurate reference calculations. The observed correlation between potential energy surfaces and dynamics is hardly surprising, but intriguingly, one might argue that lower-level ab initio calculations can still produce dynamics that yields qualitative insights into the photochemistry, especially if the shortcomings of the electronic structure calculations have been properly assessed and are considered during the analysis-but careful attention must be paid to the subtle effects which may be lost, for example in this case the switch in order between the rise of each dissociation channel. While the most important benchmark of any simulation is relevant experimental data, a particular experiment provides only a partial view of a given process. As such, in most cases it is difficult to conclusively prove or disprove the results of simulations and complementary quality measures (such as for instance evaluation of the potential energy curves) can provide important guidance on the veracity of the simulations.

In providing a detailed analysis of the $\mathrm{CS}_{2}$ dissociation dynamics, we have made extensive use of mappings of the population and structural dynamics. We particularly wish to highlight the spatio-temporal mapping of the nonadiabatic (singletsinglet and triplet-triplet) transitions, corresponding to internal conversion, as well as the singlet-triplet spin-orbit coupled transitions, corresponding to intersystem crossing, which provide interesting insight into the dynamics (such as the direct competition between IC and ISC, processes conventionally considered sequential) that is otherwise hard to disentangle from the abundant data produced by the simulations. It is worth emphasising that even in an apparently simple molecule such as $\mathrm{CS}_{2}$, remarkable complexity lies hidden in the interplay between spin-orbitcoupled electronic states and nuclear motion, a topic on which there remains much work to be done in terms of trajectorybased dynamics simulation methods.

\section{Conflicts of interest}

There are no conflicts to declare.

\section{Acknowledgements}

The authors acknowledge helpful discussions with Dr Sebastian Mai (SHARC) and Dr David Rogers (ab initio electronic structure calculations). A. K. acknowledges support from a Royal Society of Edinburgh Sabbatical Fellowship (58507) and a research grant from the Carnegie Trust for the Universities of Scotland (CRG050414). R. S. M. acknowledges funding from the Royal Society (UF100047 and UF150655). D. B. acknowledges an EPSRC studentship from the University of Edinburgh. The computational work reported used the ARCHER UK National Supercomputing Service (http://www.archer.ac.uk) and the Edinburgh Compute and Data Facility ECDF (http://www.ecdf.ed.ac.uk). 


\section{References}

1 R. S. Minns and A. Kirrander, Faraday Discuss., 2016, 194, 11-13.

2 A. Stolow, Faraday Discuss., 2013, 163, 9.

3 G. A. Worth and L. S. Cederbaum, Annu. Rev. Phys. Chem., 2004, 55, 127.

4 W. Domcke and D. R. Yarkony, Annu. Rev. Phys. Chem., 2012, 63, 325.

5 D. G. Fedorov, S. Koseki, M. W. Schmidt and M. S. Gordon, Int. Rev. Phys. Chem., 2003, 22, 551-592.

6 C. M. Marian, WIREs Comput. Mol. Sci., 2012, 2, 187-203.

7 A. R. Attar, A. Bhattacherjee, C. D. Pemmaraju, K. Schnorr, K. D. Closser, D. Prendergast and S. R. Leone, Science, 2017, 356, 54-59.

8 H. J. Wörner, J. B. Bertrand, B. Fabre, J. Higuet, H. Ruf, A. Dubrouil, S. Patchkovskii, M. Spanner, Y. Mairesse, V. Blanchet, E. Mevel, E. Constant, P. B. Corkum and D. M. Villeneuve, Science, 2011, 334, 208-212.

9 Y. Pertot, C. Schmidt, M. Matthews, A. Chauvet, M. Huppert, V. Svoboda, A. von Conta, A. Tehlar, D. Baykusheva, J.-P. Wolf and H. J. Wörner, Science, 2017, 355, 264-267.

10 A. D. Smith, E. M. Warne, D. Bellshaw, D. A. Horke, M. Tudorovskya, E. Springate, A. J. H. Jones, C. Cacho, R. T. Chapman, A. Kirrander and R. S. Minns, Phys. Rev. Lett., 2018, 120, 183003.

11 C. Z. Bisgaard, O. J. Clarkin, G. Wu, A. M. D. Lee, O. Gessner, C. C. Hayden and A. Stolow, Science, 2009, 323, 1464-1468.

12 P. Hockett, C. Z. Bisgaard, O. J. Clarkin and A. Stolow, Nat. Phys., 2011, 7, 612-615.

13 M. P. Minitti, J. M. Budarz, A. Kirrander, J. S. Robinson, D. Ratner, T. J. Lane, D. Zhu, J. M. Glownia, M. Kozina, H. T. Lemke, M. Sikorski, Y. Feng, S. Nelson, K. Saita, B. Stankus, T. Northey, J. B. Hastings and P. M. Weber, Phys. Rev. Lett., 2015, 114, 255501.

14 J. Yang, J. Beck, C. J. Uiterwaal and M. Centurion, Nat. Commun., 2015, 6, 8172.

15 T. Ishikawa, S. A. Hayes, S. Keskin, G. Corthey, M. Hada, K. Pichugin, A. Marx, J. Hirscht, K. Shionuma, K. Onda, Y. Okimoto, S.-y. Koshihara, T. Yamamoto, H. Cui, M. Nomura, Y. Oshima, M. Abdel-Jawad, R. Kato and R. J. D. Miller, Science, 2015, 350, 1501-1505.

16 M. P. Minitti, J. M. Budarz, A. Kirrander, J. Robinson, T. J. Lane, D. Ratner, K. Saita, T. Northey, B. Stankus, V. Cofer-Shabica, J. Hastings and P. M. Weber, Faraday Discuss., 2014, 171, 81.

17 C. C. Pemberton, Y. Zhang, K. Saita, A. Kirrander and P. M. Weber, J. Phys. Chem. A, 2015, 119, 8832.

18 B. Stankus, J. M. Budarz, A. Kirrander, D. Rogers, J. Robinson, T. J. Lane, D. Ratner, J. Hastings, M. P. Minitti and P. M. Weber, Faraday Discuss., 2016, 194, 525-536.

19 J. M. Budarz, M. P. Minitti, D. V. Cofer-Shabica, B. Stankus, A. Kirrander, J. B. Hastings and P. M. Weber, J. Phys. B: At., Mol. Opt. Phys., 2016, 49, 034001.

20 T. J. A. Wolf, T. S. Kuhlman, O. Schalk, T. J. Martinez, K. B. Møller, A. Stolow and A.-N. Unterreiner, Phys. Chem. Chem. Phys., 2014, 16, 11770.
21 K. Wang, V. McKoy, P. Hockett and M. S. Schuurman, Phys. Rev. Lett., 2014, 112, 113007.

22 D. V. Makhov, K. Saita, T. J. Martinez and D. V. Shalashilin, Phys. Chem. Chem. Phys., 2015, 17, 3316.

23 G. Wu, S. P. Neville, O. Schalk, T. Sekikawa, M. N. R. Ashfold, G. A. Worth and A. Stolow, J. Chem. Phys., 2015, 142, 074302.

24 M. Ruckenbauer, S. Mai, P. Marquetand and L. González, Sci. Rep., 2016, 6, 35522.

25 M. Tudorovskaya, R. S. Minns and A. Kirrander, Phys. Chem. Chem. Phys., 2018, 20, 17714-17726.

26 G. W. Richings and G. A. Worth, J. Chem. Phys., 2014, 141, 244115.

27 G. Capano, C. J. Milne, M. Chergui, U. Rothlisberger, I. Tavernelli and T. J. Penfold, J. Phys. B: At., Mol. Opt. Phys., 2015, 48, 214001.

28 A. Kirrander, K. Saita and D. V. Shalashilin, J. Chem. Theory Comput., 2016, 12, 957-967.

29 S. P. Neville, M. Chergui, A. Stolow and M. S. Schuurman, Phys. Rev. Lett., 2018, 120, 243001.

30 S. Y. Grebenshchikov, J. Chem. Phys., 2012, 137, 021101.

31 A. Kirrander, H. H. Fielding and Ch. Jungen, J. Chem. Phys., 2007, 127, 164301.

32 A. Kirrander, H. H. Fielding and Ch. Jungen, J. Chem. Phys., 2010, 132, 024313.

33 M. H. Beck, A. Jäckle, G. A. Worth and H.-D. Meyer, Phys. Rep., 2000, 324, 1-105.

34 B. G. Levine, J. D. Coe, A. M. Virshup and T. J. Martinez, Chem. Phys., 2008, 347, 3.

35 G. Worth, M. Robb and B. Lasorne, Mol. Phys., 2008, 106, 2077-2091.

36 M. Richter, P. Marquetand, J. González-Vázquez, I. Sola and L. González, J. Chem. Theory Comput., 2011, 7, 1253-1258.

37 J. C. Tully, J. Chem. Phys., 2012, 137, 22 A301.

38 M. Persico and G. Granucci, Theor. Chem. Acc., 2014, 133, 1526.

39 G. Richings, I. Polyak, K. Spinlove, G. Worth, I. Burghardt and B. Lasorne, Int. Rev. Phys. Chem., 2015, 34, 269-308.

40 D. V. Makhov, C. Symonds, S. Fernandez-Alberti and D. V. Shalashilin, Chem. Phys., 2017, 493, 200-218.

41 O. Schalk, T. Geng, T. Thompson, N. Baluyot, R. D. Thomas, E. Tapavicza and T. Hansson, J. Phys. Chem. A, 2016, 120, 2320-2329.

42 S. Mai, M. Richter, P. Marquetand and L. González, Chem. Phys., 2017, 482, 9-15.

43 R. J. Squibb, M. Sapunar, A. Ponzi, R. Richter, A. Kivimäki, O. Plekan, P. Finetti, N. Sisourat, V. Zhaunerchyk, T. Marchenko, L. Journel, R. Guillemin, R. Cucini, M. Coreno, C. Grazioli, M. D. Fraia, C. Callegari, K. C. Prince, P. Decleva, M. Simon, J. H. D. Eland, N. Doslić, R. Feifel and M. N. Piancastelli, Nat. Commun., 2018, 9, 63.

44 M. Barbatti, Z. Lan, R. Crespo-Otero, J. J. Szymczak, H. Lischka and W. Thiel, J. Chem. Phys., 2012, 137, 22 A503.

45 T. Horio, R. Spesyvtsev and T. Suzuki, Opt. Express, 2013, 21, 22423-22428.

46 R. Spesyvtsev, T. Horio, Y.-I. Suzuki and T. Suzuki, J. Chem. Phys., 2015, 142, 074308.

47 D. Bellshaw, D. A. Horke, A. D. Smith, H. M. Watts, E. Jager, E. Springate, O. Alexander, C. Cacho, R. T. Chapman, 
A. Kirrander and R. S. Minns, Chem. Phys. Lett., 2017, 683, 383-388.

48 S. Mai, M. Richter, M. Ruckenbauer, M. Oppel, P. Marquetand and L. González, SHARC: Surface Hopping Including Arbitrary Couplings - Program Package for Non-Adiabatic Dynamics, sharc-md.org, 2014.

49 S. Mai, P. Marquetand and L. González, Int. J. Quantum Chem., 2015, 115, 1215-1231.

50 J. C. Tully, J. Chem. Phys., 1990, 93, 1061-1071.

51 S. P. McGlynn, J. W. Rabalais, J. R. McDonald and V. Scherr, Chem. Rev., 1971, 71, 73-108.

52 S. Ahmed and V. Kumar, Pramana, 1992, 39, 367-380.

53 L. M. Goss, G. J. Frost, D. Donaldson and V. Vaida, Geophys. Res. Lett., 1995, 22, 2609-2612.

54 H.-J. Werner, P. J. Knowles, G. Knizia, F. R. Manby and M. Schütz et al., MOLPRO, version 2012.1, a package of ab initio programs.
55 Q. Zhang and P. H. Vaccaro, J. Phys. Chem., 1995, 99, 1799-1813.

56 F. Plasser, M. Ruckenbauer, S. Mai, M. Oppel, P. Marquetand and L. González, J. Chem. Theory Comput., 2016, 12, 1207-1219.

57 G. Granucci, M. Persico and A. Zoccante, J. Chem. Phys., 2010, 133, 134111.

58 M. Barbatti, M. Ruckenbauer, F. Plasser, J. Pittner, G. Granucci, M. Persico and H. Lischka, Wiley Interdiscip. Rev.: Comput. Mol. Sci., 2014, 4, 26-33.

59 B. O. Roos, Adv. Chem. Phys., 1987, 69, 399-445.

60 D. M. Rogers, C. Wells, M. Joseph, V. J. Boddington and J. J. McDouall, J. Mol. Struct.: THEOCHEM, 1998, 434, 239-245.

61 V. Veryazov, P. Å. Malmqvist and B. O. Roos, Int. J. Quantum Chem., 2011, 111, 3329-3338.

62 T. Trabelsi, M. M. Al-Mogren, M. Jochlaf and J. S. Francisco, J. Chem. Phys., 2018, 149, 064304. 